\title{
Nonlinear stability of periodic traveling wave solutions of viscous conservation laws in dimensions one and two
}

\author{
Mathew A. Johnson* Kevin Zumbrun ${ }^{\dagger}$
}

November 12, 2018

Keywords: Periodic traveling waves; Bloch decomposition; modulated waves.

2000 MR Subject Classification: 35B35.

\begin{abstract}
Extending results of $\mathrm{Oh}$ and Zumbrun in dimensions $d \geq 3$, we establish nonlinear stability and asymptotic behavior of spatially-periodic traveling-wave solutions of viscous systems of conservation laws in critical dimensions $d=1,2$, under a natural set of spectral stability assumptions introduced by Schneider in the setting of reaction diffusion equations. The key new steps in the analysis beyond that in dimensions $d \geq 3$ are a refined Green function estimate separating off translation as the slowest decaying linear mode and a novel scheme for detecting cancellation at the level of the nonlinear iteration in the Duhamel representation of a modulated periodic wave.
\end{abstract}

\section{Introduction}

Nonclassical viscous conservation laws arising in multiphase fluid and solid mechanics exhibit a rich variety of traveling wave phenomena, including homoclinic (pulse-type) and periodic solutions along with the standard heteroclinic (shock, or front-type) solutions GZ, Z6, OZ1, OZ2. Here, we investigate stability of periodic traveling waves: specifically, sufficient conditions for stability of the wave. Our main result, generalizing results of Oh and Zumbrun OZ4 in dimensions $d \geq 3$, is to show that strong spectral stability in the sense of Schneider [S1, S2, [S3] implies linearized and nonlinear $L^{1} \cap H^{K} \rightarrow L^{\infty}$ bounded stability, for all dimensions $d \geq 1$, and asymptotic stability for dimensions $d \geq 2$.

\footnotetext{
*Indiana University, Bloomington, IN 47405; matjohn@indiana.edu: Research of M.J. was partially supported by an NSF Postdoctoral Fellowship under NSF grant DMS-0902192.

${ }^{\dagger}$ Indiana University, Bloomington, IN 47405; kzumbrun@indiana.edu: Research of K.Z. was partially supported under NSF grants no. DMS-0300487 and DMS-0801745.
} 
More precisely, we show that small $L^{1} \cap H^{s}$ perturbations of a planar periodic solution $u(x, t) \equiv \bar{u}\left(x_{1}\right)$ (without loss of generality taken stationary) converge at Gaussian rate in $L^{p}, p \geq 2$ to a modulation

$$
\bar{u}\left(x_{1}-\psi(x, t)\right)
$$

of the unperturbed wave, where $x=\left(x_{1}, \tilde{x}\right), \tilde{x}=\left(x_{2}, \ldots, x_{d}\right)$, and $\psi$ is a scalar function whose $x$ - and $t$-gradients likewise decay at least at Gaussian rate in all $L^{p}, p \geq 2$, but which itself decays more slowly by a factor $t^{1 / 2}$; in particular, $\psi$ is merely bounded in $L^{\infty}$ for dimension $d=1$.

The one-dimensional study of spectral stability of spatially periodic traveling waves of systems of viscous conservation laws was initiated by Oh and Zumbrun [OZ1] in the "quasiHamiltonian" case that the traveling-wave equation possesses an integral of motion, and in the general case by Serre [Se1]. An important contribution of Serre was to point out a larger connection between the linearized dispersion relation (the function $\lambda(\xi)$ relating spectra to wave number of the linearized operator about the wave) near zero and the formal Whitham averaged system obtained by slow modulation, or WKB, approximation.

In [OZ3, this was extended to multi-dimensions, relating the linearized dispersion relation near zero to

$$
\begin{gathered}
\partial_{t} M+\sum_{j} \partial_{x_{j}} F^{j}=0, \\
\partial_{t}(\Omega N)+\nabla_{x}(\Omega S)=0,
\end{gathered}
$$

where $M \in \mathbb{R}^{n}$ denotes the average over one period, $F^{j}$ the average of an associated flux, $\Omega=\left|\nabla_{x} \Psi\right| \in \mathbb{R}^{1}$ the frequency, $S=-\Psi_{t} /\left|\nabla_{x} \Psi\right| \in \mathbb{R}^{1}$ the speed $s$, and $N=\nabla_{x} \Psi /\left|\nabla_{x} \Psi\right| \in$ $\mathbb{R}^{d}$ the normal $\nu$ associated with nearby periodic waves, with an additional constraint

$$
\operatorname{curl}(\Omega N)=\operatorname{curl} \nabla_{x} \Psi \equiv 0 .
$$

As an immediate corollary, similarly as in [OZ1, [Se1] in the one-dimensional case, this yielded as a necessary condition for multi-dimensional stability hyperbolicity of the averaged system (1.2)-(1.3).

The present study is informed by but does not directly rely on this observation relating Whitham averaging and spectral stability properties. Likewise, the Evans function techniques used in [Se1, OZ3] to establish this connection play no role in our analysis; indeed, the Evans function makes no appearance here. Rather, we rely on a direct Bloch-decomposition argument in the spirit of Schneider [S1, S2, S3], combining sharp linearized estimates with subtle cancellation in nonlinear source terms arising from the modulated wave approximation. The analytical techniques used to realize this program are somewhat different from those of [S1, S2, S3], however, coming instead from the theory of stability of viscous shock fronts through a line of investigation carried out in [OZ1, OZ2, OZ3, OZ4, HoZ]. In particular, the nonsmooth dispersion relation at $\xi=0$ typical for convection-diffusion equations 
requires different treatment from that of $[\mathrm{S} 1, \mathrm{S2}$, $\mathrm{S} 3]$ in the reaction diffusion case; see Remark 2.4. Moreover, we detect nonlinear cancellation in the physical $x$ - $t$ domain rather than the frequency domain as in [S1, S2, S3]. The main difference between the present analysis and that of [OZ4] is the systematic incorporation of modulation approximation (1.1).

\subsection{Equations and assumptions}

Consider a parabolic system of conservation laws

$$
u_{t}+\sum_{j} f^{j}(u)_{x_{j}}=\Delta_{x} u
$$

$u \in \mathcal{U}$ (open) $\in \mathbb{R}^{n}, f^{j} \in \mathbb{R}^{n}, x \in \mathbb{R}^{d}, d \geq 1, t \in \mathbb{R}^{+}$, and a periodic traveling wave solution

$$
u=\bar{u}(x \cdot \nu-s t)
$$

of period $X$, satisfying the traveling-wave ODE $\bar{u}^{\prime \prime}=\left(\sum_{j} \nu_{j} f^{j}(\bar{u})\right)^{\prime}-s \bar{u}^{\prime}$ with boundary conditions $\bar{u}(0)=\bar{u}(X)=: u_{0}$. Integrating, we obtain a first-order profile equation

$$
\bar{u}^{\prime}=\sum_{j} \nu_{j} f^{j}(\bar{u})-s \bar{u}-q,
$$

where $\left(u_{0}, q, s, \nu, X\right) \equiv$ constant. Without loss of generality take $\nu=e_{1}, s=0$, so that $\bar{u}=\bar{u}\left(x_{1}\right)$ represents a stationary solution depending only on $x_{1}$.

Following [Se1, OZ3, OZ4, we assume:

(H1) $f^{j} \in C^{K+1}, K \geq[d / 2]+4$.

(H2) The map $H: \mathbb{R} \times \mathcal{U} \times \mathbb{R} \times S^{d-1} \times \mathbb{R}^{n} \rightarrow \mathbb{R}^{n}$ taking $(X ; a, s, \nu, q) \mapsto u(X ; a, s, \nu, q)-a$ is a submersion at point $\left(\bar{X} ; \bar{u}(0), 0, e_{1}, \bar{q}\right)$, where $u(\cdot ; \cdot)$ is the solution operator for (1.6).

Conditions (H1)-(H2) imply that the set of periodic solutions in the vicinity of $\bar{u}$ form a smooth $(n+d+1)$-dimensional manifold $\left\{\bar{u}^{a}(x \cdot \nu(a)-\alpha-s(a) t)\right\}$, with $\alpha \in \mathbb{R}, a \in \mathbb{R}^{n+d}$.

\subsubsection{Linearized equations}

Linearizing (1.4) about $\bar{u}(\cdot)$, we obtain

$$
v_{t}=L v:=\Delta_{x} v-\sum\left(A^{j} v\right)_{x_{j}}
$$

where coefficients $A^{j}:=D f^{j}(\bar{u})$ are now periodic functions of $x_{1}$. Taking the Fourier transform in the transverse coordinate $\tilde{x}=\left(x_{2}, \cdots, x_{d}\right)$, we obtain

$$
\hat{v}_{t}=L_{\tilde{\xi}} \hat{v}=\hat{v}_{x_{1}, x_{1}}-\left(A^{1} \hat{v}\right)_{x_{1}}-i \sum_{j \neq 1} A^{j} \xi_{j} \hat{v}-\sum_{j \neq 1} \xi_{j}^{2} \hat{v},
$$

where $\tilde{\xi}=\left(\xi_{2}, \cdots, \xi_{d}\right)$ is the transverse frequency vector. 


\subsubsection{Bloch-Fourier decomposition and stability conditions}

Following [G, S1, S2, S3, we define the family of operators

$$
L_{\xi}=e^{-i \xi_{1} x_{1}} L_{\tilde{\xi}} e^{i \xi_{1} x_{1}}
$$

operating on the class of $L^{2}$ periodic functions on $[0, X]$; the $\left(L^{2}\right)$ spectrum of $L_{\tilde{\xi}}$ is equal to the union of the spectra of all $L_{\xi}$ with $\xi_{1}$ real with associated eigenfunctions

$$
w\left(x_{1}, \tilde{\xi}, \lambda\right):=e^{i \xi_{1} x_{1}} q\left(x_{1}, \xi_{1}, \tilde{\xi}, \lambda\right)
$$

where $q$, periodic, is an eigenfunction of $L_{\xi}$. By continuity of spectrum, and discreteness of the spectrum of the elliptic operators $L_{\xi}$ on the compact domain $[0, X]$, we have that the spectra of $L_{\xi}$ may be described as the union of countably many continuous surfaces $\lambda_{j}(\xi)$.

Without loss of generality taking $X=1$, recall now the Bloch-Fourier representation

$$
u(x)=\left(\frac{1}{2 \pi}\right)^{d} \int_{-\pi}^{\pi} \int_{\mathbb{R}^{d-1}} e^{i \xi \cdot x} \hat{u}\left(\xi, x_{1}\right) d \xi_{1} d \tilde{\xi}
$$

of an $L^{2}$ function $u$, where $\hat{u}\left(\xi, x_{1}\right):=\sum_{k} e^{2 \pi i k x_{1}} \hat{u}\left(\xi_{1}+2 \pi k, \tilde{\xi}\right)$ are periodic functions of period $X=1, \hat{u}(\tilde{\xi})$ denoting with slight abuse of notation the Fourier transform of $u$ in the full variable $x$. By Parseval's identity, the Bloch-Fourier transform $u(x) \rightarrow \hat{u}\left(\xi, x_{1}\right)$ is an isometry in $L^{2}$ :

$$
\|u\|_{L^{2}(x)}=\|\hat{u}\|_{L^{2}\left(\xi ; L^{2}\left(x_{1}\right)\right)},
$$

where $L^{2}\left(x_{1}\right)$ is taken on $[0,1]$ and $L^{2}(\xi)$ on $[-\pi, \pi] \times \mathbb{R}^{d-1}$. Moreover, it diagonalizes the periodic-coefficient operator $L$, yielding the inverse Bloch-Fourier transform representation

$$
e^{L t} u_{0}=\left(\frac{1}{2 \pi}\right)^{d} \int_{-\pi}^{\pi} \int_{\mathbb{R}^{d-1}} e^{i \xi \cdot x} e^{L_{\xi} t} \hat{u}_{0}\left(\xi, x_{1}\right) d \xi_{1} d \tilde{\xi}
$$

relating behavior of the linearized system to that of the diagonal operators $L_{\xi}$.

Following [OZ4, we assume along with (H1)-(H2) the strong spectral stability conditions:

(D1) $\sigma\left(L_{\xi}\right) \subset\{\operatorname{Re} \lambda<0\}$ for $\xi \neq 0$.

(D2) $\operatorname{Re} \sigma\left(L_{\xi}\right) \leq-\theta|\xi|^{2}, \theta>0$, for $\xi \in \mathbb{R}^{d}$ and $|\xi|$ sufficiently small.

(D3) $\lambda=0$ is a semisimple eigenvalue of $L_{0}$ of multiplicity exactly $n+11$

For each fixed angle $\hat{\xi}:=\xi /|\xi|$, expand $L_{\xi}=L_{0}+|\xi| L^{1}+|\xi|^{2} L^{2}$. By assumption (D3) and standard spectral perturbation theory, there exist $n+1$ smooth eigenvalues

$$
\lambda_{j}(\xi)=-i a_{j}(\xi)+o(|\xi|)
$$

\footnotetext{
${ }^{1}$ The zero eigenspace of $L_{0}$ is at least $(n+1)$-dimensional by the linearized existence theory and $(\mathrm{H} 2)$, and hence $n+1$ is the minimal multiplicity; see [Se1, OZ3. As noted in OZ1, OZ3, minimal dimension of this zero eigenspace implies that $(M, N \Omega)$ of (1.2) gives a nonsingular coordinatization of the family of periodic traveling-wave solutions near $\bar{u}$.
} 
of $L_{\xi}$ bifurcating from $\lambda=0$ at $\xi=0$, where $-i a_{j}$ are homogeneous degree one functions given by $|\xi|$ times the eigenvalues of $\left.\Pi_{0} L^{1}\right|_{\operatorname{Ker} L_{0}}$, with $\Pi_{0}$ the zero eigenprojection of $L_{0}$.

Conditions (D1)-(D3) are exactly the spectral assumptions of [S1, S2, S3], corresponding to "dissipativity" of the large-time behavior of the linearized system. As in [OZ4], we make the further nondegeneracy hypothesis:

(H3) The eigenvalues $\lambda=-i a_{j}(\xi) /|\xi|$ of $\Pi_{0} L_{\operatorname{Ker} L_{0}}^{1}$ are simple.

The functions $a_{j}$ may be seen to be the characteristics associated with the Whitham averaged system (1.2)-(1.3) linearized about the values of $M, S, N, \Omega$ associated with the background wave $\bar{u}$; see [OZ3, OZ4]. Thus, (D1) implies weak hyperbolicity of (1.2)-(1.3) (reality of $a_{j}$ ), while (H1) corresponds to strict hyperbolicity.

\subsection{Main results}

With these preliminaries, we can now state our main results.

Theorem 1.1. Assuming (H1)-(H3) and (D1)-(D3), for some $C>0$ and $\psi \in W^{K, \infty}(x, t)$,

$$
\begin{aligned}
& |\tilde{u}-\bar{u}(\cdot-\psi)|_{L^{p}}(t) \leq\left. C(1+t)^{-\frac{d}{2}(1-1 / p)}|\tilde{u}-\bar{u}|_{L^{1} \cap H^{K}}\right|_{t=0}, \\
& |\tilde{u}-\bar{u}(\cdot-\psi)|_{H^{K}}(t) \leq\left. C(1+t)^{-\frac{d}{4}}|\tilde{u}-\bar{u}|_{L^{1} \cap H^{K}}\right|_{t=0}, \\
& \left|\left(\psi_{t}, \psi_{x}\right)\right|_{W^{K+1, p}} \leq\left. C(1+t)^{-\frac{d}{2}(1-1 / p)}|\tilde{u}-\bar{u}|_{L^{1} \cap H^{K}}\right|_{t=0},
\end{aligned}
$$

and

$$
|\tilde{u}-\bar{u}|_{L^{p}}(t),|\psi(t)|_{L^{p}} \leq\left. C(1+t)^{-\frac{d}{2}\left(1-\frac{1}{p}\right)+\frac{1}{2}}|\tilde{u}-\bar{u}|_{L^{1} \cap H^{K}}\right|_{t=0}
$$

for all $t \geq 0, p \geq 2, d=1$, for solutions $\tilde{u}$ of (1.4) with $\left.|\tilde{u}-\bar{u}|_{L^{1} \cap H^{K}}\right|_{t=0}$ sufficiently small. In particular, $\bar{u}$ is nonlinearly bounded $L^{1} \cap H^{K} \rightarrow L^{\infty}$ stable for dimension $d=1$.

Theorem 1.2. Assuming (H1)-(H3) and (D1)-(D3), for any $\varepsilon>0$, some $C>0$ and $\psi \in W^{K, \infty}(x, t)$,

$$
\begin{aligned}
& |\tilde{u}-\bar{u}(\cdot-\psi)|_{L^{p}}(t) \leq\left. C(1+t)^{-\frac{d}{2}(1-1 / p)}|\tilde{u}-\bar{u}|_{L^{1} \cap H^{K}}\right|_{t=0}, \\
& |\tilde{u}-\bar{u}(\cdot-\psi)|_{H^{K}}(t) \leq\left. C(1+t)^{-\frac{d}{4}}|\tilde{u}-\bar{u}|_{L^{1} \cap H^{K}}\right|_{t=0}, \\
& \left|\left(\psi_{t}, \psi_{x}\right)\right|_{W^{K+1, p}} \leq\left. C(1+t)^{-\frac{d}{2}(1-1 / p)+\varepsilon-\frac{1}{2}}|\tilde{u}-\bar{u}|_{L^{1} \cap H^{K}}\right|_{t=0},
\end{aligned}
$$

and

$$
\begin{aligned}
|\tilde{u}-\bar{u}|_{L^{p}}(t),|\psi(t)|_{L^{p}} & \leq\left. C(1+t)^{-\frac{d}{2}\left(1-\frac{1}{p}\right)+\varepsilon}|\tilde{u}-\bar{u}|_{L^{1} \cap H^{K}}\right|_{t=0}, \\
|\tilde{u}-\bar{u}|_{H^{K}}(t),|\psi(t)|_{H^{K}} & \leq\left. C(1+t)^{-\frac{d}{4}+\varepsilon}|\tilde{u}-\bar{u}|_{L^{1} \cap H^{K}}\right|_{t=0},
\end{aligned}
$$

for all $t \geq 0, p \geq 2, d=2$, for solutions $\tilde{u}$ of (1.4) with $\left.|\tilde{u}-\bar{u}|_{L^{1} \cap H^{K}}\right|_{t=0}$ sufficiently small. In particular, $\bar{u}$ is nonlinearly asymptotically $L^{1} \cap H^{K} \rightarrow H^{K}$ stable for dimension $d=2$. 
Remark 1.1. In Theorem 1.2, derivatives in $x \in \mathbb{R}^{2}$ refer to total derivatives. Moreover, unless specified by an appropriate index, throughout this paper derivatives in spatial variable $x$ will always refer to the total derivative of the function.

In dimension one, Theorem 1.1 asserts only bounded $L^{1} \cap H^{K} \rightarrow L^{\infty}$ stability, a very weak notion of stability. The absence of decay in perturbation $\tilde{u}-\bar{u}$ indicates the delicacy of the nonlinear analysis in this case. In particular, it is crucial to separate off the slowerdecaying modulated behavior (1.1) in order to close the nonlinear iteration argument.

Remark 1.2. In dimension $d=1$, it is straightforward to show that the results of Theorem 1.1 extend to all $1 \leq p \leq \infty$ using the pointwise techniques of OZ2, see Remark 3.3.

Remark 1.3. The slow decay of $|\tilde{u}-\bar{u}|_{L^{p}}(t) \sim|\psi(t)|_{L^{p}}$ in (1.16) is due to nonlinear interactions; as shown in [OZ2, OZ4], the linearized decay rate is faster by factor $(1+t)^{-1 / 2}$ (Proposition 2.1). In [OZ4, it was shown that for $d \geq 3$, where linear effects dominate behavior, (1.16) may be replaced by the stronger estimate $|\tilde{u}-\bar{u}|_{L^{p}}(t),|\psi(t)|_{L^{p}} \leq C(1+$

$t)\left.^{-\frac{d}{2}\left(1-\frac{1}{p}\right)}|\tilde{u}-\bar{u}|_{L^{1} \cap H^{K}}\right|_{t=0}$. These distinctions reflect fine details of both linearized estimates (Section 3) and nonlinear structure (Sections 4.14.2) that are not immediately apparent from the formal Whitham approximation (1.2)-(1.3).

\subsection{Discussion and open problems}

Linearized stability under the same assumptions, with sharp rates of decay, was established for $d=1$ [OZ2] and for $d \geq 1$ in [OZ4], along with nonlinear stability for $d \geq 3$. Theorem 1.1 completes this line of investigation by establishing nonlinear stability in the critical dimensions $d=1,2$, a fundamental open problem cited in [OZ1, OZ4].

This gives a generalization of the work of [S1, S2, S3] for reaction diffusion equations to the case of viscous conservation laws. Recall that the analysis of [S1, S2, S3] concerns also multiply periodic waves, i.e., waves that are either periodic or else constant in each coordinate direction. It is straightforward to verify that the methods of this paper apply essentially unchanged to this case, to give a corresponding stability result under the analog of (H1)-(H3), (D1)-(D3), as we intend to report further in a future work. Likewise, the extension from the semilinear parabolic case treated here to the general quasilinear case is straightforward, following the treatment of OZ4.

On the other hand, as noted in OZ2, condition (D3) is in the conservation law setting nongeneric, corresponding to the special "quasi-Hamiltonian" situation studied there; in particular, it implies that speed is to first order constant among the family of spatially periodic traveling-wave solutions nearby $\bar{u}$. In the generic case that (D3) is violated, behavior is essentially different [OZ1, OZ2], and perturbations decay more slowly at the linearized level. Nonlinear stability remains an interesting open problem in this setting.

Our approach to stability in the critical dimensions $d=1,2$, as suggested in [OZ4], is, loosely following the approach of [S1, S2, [S3], to subtract out a slower-decaying part of the solution described by an appropriate modulation equation and show that the residual decays 
sufficiently rapidly to close a nonlinear iteration. It is worth noting that the modulated approximation $\bar{u}\left(x_{1}-\psi(x, t)\right)$ of (1.1) is not the full Ansatz

$$
\bar{u}^{a}(\Psi(x, t))
$$

$\Psi(x, t):=x_{1}-\psi(x, t)$, associated with the Whitham averaged system (1.2)-(1.3), where $\bar{u}^{a}$ is the manifold of periodic solutions near $\bar{u}$ introduced below (H2), but only the translational part not involving perturbations $a$ in the profile. (See OZ3] for the derivation of (1.2)(1.3) and (1.19).) That is, we don't need to separate out all variations along the manifold of periodic solutions, but only the special variations connected with translation invariance.

The technical reason is an asymmetry in $y$-derivative estimates in the parts of the Green function associated with these various modes, something that is not apparent without a detailed study of linearized behavior as carried out here. This also makes sense formally, if one considers that (1.2) indicates that variables $a, \nabla_{x} \Psi$ are roughly comparable, which would suggest, by the diffusive behavior $\Psi>>\nabla_{x} \Psi$, that $a$ is neglible with respect to $\Psi$.

However, note that in the case that (D3) holds, hence wave speed is stationary along the manifold of periodic solutions, the final equation of (1.2) decouples to $\left(\Psi_{x}\right)_{t}=(\Omega N)_{t}=0$, and could be written as $\Psi_{t}=0$ in terms of $\Psi$ alone. Hence, there is some ambiguity in this degenerate case which of $\Psi, \Psi_{x}$ is the primary variable, and in terms of linear behavior, the decay of variations $a$ and $\Psi$ are in fact comparable [OZ4; in the generic case, $a$ and $\Psi_{x}$ are comparable at the linearized level [OZ2]. It would be very interesting to better understand the connection between the Whitham averaged system (or suitable higher-order correction) and behavior at the nonlinear level, as explored at the linear level in [OZ3, OZ4, JZ1, JZB].

\section{Basic linearized stability estimates}

We begin by recalling the basic linearized stability estimates derived in OZ4. We will sharpen these afterward in Section 3. By standard spectral perturbation theory $[\mathrm{K}]$, the total eigenprojection $P(\xi)$ onto the eigenspace of $L_{\xi}$ associated with the eigenvalues $\lambda_{j}(\xi)$, $j=1, \ldots, n+1$ described in the introduction is well-defined and analytic in $\xi$ for $\xi$ sufficiently small, since these (by discreteness of the spectra of $L_{\xi}$ ) are separated at $\xi=0$ from the rest of the spectrum of $L_{0}$. Introducing a smooth cutoff function $\phi(\xi)$ that is identically one for $|\xi| \leq \varepsilon$ and identically zero for $|\xi| \geq 2 \varepsilon, \varepsilon>0$ sufficiently small, we split the solution operator $S(t):=e^{L t}$ into low- and high-frequency parts

$$
S^{I}(t) u_{0}:=\left(\frac{1}{2 \pi}\right)^{d} \int_{-\pi}^{\pi} \int_{\mathbb{R}^{d-1}} e^{i \xi \cdot x} \phi(\xi) P(\xi) e^{L_{\xi} t} \hat{u}_{0}\left(\xi, x_{1}\right) d \xi_{1} d \tilde{\xi}
$$

and

$$
S^{I I}(t) u_{0}:=\left(\frac{1}{2 \pi}\right)^{d} \int_{-\pi}^{\pi} \int_{\mathbb{R}^{d-1}} e^{i \xi \cdot x}(I-\phi P(\xi)) e^{L_{\xi} t} \hat{u}_{0}\left(\xi, x_{1}\right) d \xi_{1} d \tilde{\xi}
$$




\subsection{High-frequency bounds}

By standard sectorial bounds $[\mathrm{He}, \mathrm{Pa}]$ and spectral separation of $\lambda_{j}(\xi)$ from the remaining spectra of $L_{\xi}$, we have trivially the exponential decay bounds

$$
\begin{aligned}
\left\|e^{L_{\xi} t}(I-\phi P(\xi)) f\right\|_{L^{2}([0, X])} & \leq C e^{-\theta t}\|f\|_{L^{2}([0, X])}, \\
\left\|e^{L_{\xi} t}(I-\phi P(\xi)) \partial_{x_{1}}^{l} f\right\|_{L^{2}([0, X])} & \leq C t^{-\frac{l}{2}} e^{-\theta t}\|f\|_{L^{2}([0, X])}, \\
\left\|\partial_{x_{1}}^{l} e^{L_{\xi} t}(I-\phi P(\xi)) f\right\|_{L^{2}([0, X])} & \leq C t^{-\frac{l}{2}} e^{-\theta t}\|f\|_{L^{2}([0, X])},
\end{aligned}
$$

for $\theta, C>0$, and $0 \leq m \leq K(K$ as in (H1)). Together with (1.12), these give immediately the following estimates.

Proposition 2.1 ([OZ4). Under assumptions (H1)-(H3) and (D1)-(D2), for some $\theta$, $C>0$, and all $t>0,2 \leq p \leq \infty, 0 \leq l \leq K+1,0 \leq m \leq K$,

$$
\begin{aligned}
\left\|\partial_{x}^{l} S^{I I}(t) f\right\|_{L^{2}(x)},\left\|S^{I I}(t) \partial_{x}^{l} f\right\|_{L^{2}(x)} & \leq C t^{-\frac{l}{2}} e^{-\theta t}\|f\|_{L^{2}(x)} \\
\left\|\partial_{x}^{m} S^{I I}(t) f\right\|_{L^{p}(x)},\left\|S^{I I}(t) \partial_{x}^{m} f\right\|_{L^{p}(x)} & \leq C t^{-\frac{d}{2}\left(\frac{1}{2}-\frac{1}{p}\right)-\frac{m}{2}} e^{-\theta t}\|f\|_{L^{2}(x)}
\end{aligned}
$$

where, again, derivatives in the variable $x \in \mathbb{R}^{d}$ refer to total derivatives.

Proof. The first inequalities follow immediately by (1.12) and (2.3). The second follows for $x_{1}$ derivatives in the case $p=\infty, m=0$ by Sobolev embedding from

$$
\left\|S^{I I}(t) f\right\|_{L^{\infty}\left(\tilde{x} ; L^{2}\left(x_{1}\right)\right)} \leq C t^{-\frac{d-1}{4}} e^{-\theta t}\|f\|_{L^{2}([0, X])}
$$

and

$$
\left\|\partial_{x_{1}} S^{I I}(t) f\right\|_{L^{\infty}\left(\tilde{x} ; L^{2}\left(x_{1}\right)\right)} \leq C t^{-\frac{d-1}{4}-\frac{1}{2}} e^{-\theta t}\|f\|_{L^{2}([0, X])},
$$

which follow by an application of (1.12) in the $x_{1}$ variable and the Hausdorff-Young inequality $\|f\|_{L^{\infty}(\tilde{x})} \leq\|\hat{f}\|_{L^{1}(\tilde{\xi})}$ in the variable $\tilde{x}$. The result for derivatives in $x_{1}$ and general $2 \leq p \leq \infty$ then follows by $L^{p}$ interpolation. Finally, the result for derivatives in $\tilde{x}$ follows from the inverse Fourier transform, equation (2.2), and the large $|\xi|$ bound

$$
\left|e^{L t} f\right|_{L^{2}\left(x_{1}\right)} \leq e^{-\theta|\tilde{\xi}|^{2} t}|f|_{L^{2}\left(x_{1}\right)},|\xi| \text { sufficiently large, }
$$

which easily follows from Parseval and the fact that $L_{\xi}$ is a relatively compact perturbation of $\partial_{x}^{2}-|\xi|^{2}$. Thus, by the above estimate we have

$$
\begin{aligned}
\left\|e^{L t} \partial_{\tilde{x}} f\right\|_{L^{2}(x)} & \leq C\left\|e^{L_{\xi} t}|\tilde{\xi}| \hat{f}\right\|_{L^{2}\left(x_{1}, \xi\right)} \\
& \leq C \sup \left(e^{-\theta|\tilde{\xi}|^{2} t}|\xi|\right)\|\hat{f}\|_{L^{2}\left(x_{1}, \xi\right)} \\
& \leq C t^{-1 / 2}\|f\|_{L^{2}(x)} .
\end{aligned}
$$

A similar argument applies for $1 \leq m \leq K$. 


\subsection{Low-frequency bounds}

Denote by

$$
G^{I}(x, t ; y):=S^{I}(t) \delta_{y}(x)
$$

the Green kernel associated with $S^{I}$, and

$$
\left[G_{\xi}^{I}\left(x_{1}, t ; y_{1}\right)\right]:=\phi(\xi) P(\xi) e^{L_{\xi} t}\left[\delta_{y_{1}}\left(x_{1}\right)\right]
$$

the corresponding kernel appearing within the Bloch-Fourier representation of $G^{I}$, where the brackets on $\left[G_{\xi}\right]$ and $\left[\delta_{y}\right]$ denote the periodic extensions of these functions onto the whole line. Then, we have the following descriptions of $G^{I},\left[G_{\xi}^{I}\right]$, deriving from the spectral expansion (1.14) of $L_{\xi}$ near $\xi=0$.

Proposition 2.2 ([OZ4]). Under assumptions (H1)-(H3) and (D1)-(D3),

$$
\begin{aligned}
{\left[G_{\xi}^{I}\left(x_{1}, t ; y_{1}\right)\right] } & =\phi(\xi) \sum_{j=1}^{n+1} e^{\lambda_{j}(\xi) t} q_{j}\left(\xi, x_{1}\right) \tilde{q}_{j}\left(\xi, y_{1}\right)^{*}, \\
G^{I}(x, t ; y) & =\left(\frac{1}{2 \pi}\right)^{d} \int_{\mathbb{R}^{d}} e^{i \xi \cdot(x-y)}\left[G_{\xi}^{I}\left(x_{1}, t ; y_{1}\right)\right] d \xi \\
& =\left(\frac{1}{2 \pi}\right)^{d} \int_{\mathbb{R}^{d}} e^{i \xi \cdot(x-y)} \phi(\xi) \sum_{j=1}^{n+1} e^{\lambda_{j}(\xi) t} q_{j}\left(\xi, x_{1}\right) \tilde{q}_{j}\left(\xi, y_{1}\right)^{*} d \xi,
\end{aligned}
$$

where $*$ denotes matrix adjoint, or complex conjugate transpose, $q_{j}(\xi, \cdot)$ and $\tilde{q}_{j}(\xi, \cdot)$ are right and left eigenfunctions of $L_{\xi}$ associated with eigenvalues $\lambda_{j}(\xi)$ defined in (1.14), normalized so that $\left\langle\tilde{q}_{j}, q_{j}\right\rangle \equiv 1$, where $\lambda_{j} /|\xi|$ is a smooth function of $|\xi|$ and $\hat{\xi}:=\xi /|\xi|$ and $q_{j}$ and $\tilde{q}_{j}$ are smooth functions of $|\xi|, \hat{\xi}:=\xi /|\xi|$, and $x_{1}$ or $y_{1}$, with $\Re \lambda_{j}(\xi) \leq-\theta|\xi|^{2}$.

Proof. Smooth dependence of $\lambda_{j}$ and of $q, \tilde{q}$ as functions in $L^{2}[0, X]$ follow from standard spectral perturbation theory $[\mathrm{K}]$ using the fact that $\lambda_{j}$ split to first order in $|\xi|$ as $\xi$ is varied along rays through the origin, and that $L_{\xi}$ varies smoothly with angle $\hat{\xi}$. Smoothness of $q_{j}, \tilde{q}_{j}$ in $x_{1}, y_{1}$ then follow from the fact that they satisfy the eigenvalue equation for $L_{\xi}$, which has smooth, periodic coefficients. Likewise, (2.7)(i) is immediate from the spectral decomposition of elliptic operators on finite domains. Substituting (2.5) into (2.1) and computing

$$
\widehat{\delta_{y}}\left(\xi, x_{1}\right)=\sum_{k} e^{2 \pi i k x_{1}} \widehat{\delta_{y}}\left(\xi+2 \pi k e_{1}\right)=\sum_{k} e^{2 \pi i k x_{1}} e^{-i \xi \cdot y-2 \pi i k y_{1}}=e^{-i \xi \cdot y}\left[\delta_{y_{1}}\left(x_{1}\right)\right],
$$

where the second and third equalities follow from the fact that the Fourier transform either continuous or discrete of the delta-function is unity, we obtain

$$
\begin{aligned}
G^{I}(x, t ; y) & =\left(\frac{1}{2 \pi}\right)^{d} \int_{-\pi}^{\pi} \int_{\mathbb{R}^{d-1}} e^{i \xi \cdot x} \phi P(\xi) e^{L_{\xi} t} \widehat{\delta}_{y}\left(\xi, x_{1}\right) d \xi \\
& =\left(\frac{1}{2 \pi}\right)^{d} \int_{-\pi}^{\pi} \int_{\mathbb{R}^{d-1}} e^{i \xi \cdot(x-y)} \phi P(\xi) e^{L_{\xi} t}\left[\delta_{y_{1}}\left(x_{1}\right)\right] d \xi
\end{aligned}
$$


yielding (2.7) (ii) by (2.6) (i) and the fact that $\phi$ is supported on $[-\pi, \pi]$.

Proposition 2.3 ([OZ4]). Under assumptions (H1)-(H3) and (D1)-(D3),

$$
\sup _{y}\left\|G^{I}(\cdot, t, ; y)\right\|_{L^{p}(x)}, \sup _{y}\left\|\partial_{x, y} G^{I}(\cdot, t, ; y)\right\|_{L^{p}(x)} \leq C(1+t)^{-\frac{d}{2}\left(1-\frac{1}{p}\right)}
$$

for all $2 \leq p \leq \infty, t \geq 0$, where $C>0$ is independent of $p$.

Proof. From representation (2.7)(ii) and $\Re \lambda_{j}(\xi) \leq-\theta|\xi|^{2}$, we obtain by the triangle inequality

$$
\left\|G^{I}\right\|_{L^{\infty}(x, y)} \leq C\left\|e^{-\theta|\xi|^{2} t} \phi(\xi)\right\|_{L^{1}(\xi)} \leq C(1+t)^{-\frac{d}{2}},
$$

verifying the bounds for $p=\infty$. Derivative bounds follow similarly, since derivatives falling on $q_{j}$ or $\tilde{q}_{j}$ are harmless, whereas derivatives falling on $e^{i \xi \cdot(x-y)}$ bring down a factor of $\xi$, again harmless because of the cutoff function $\phi$.

To obtain bounds for $p=2$, we note that (2.7) (ii) may be viewed itself as a BlochFourier decomposition with respect to variable $z:=x-y$, with $y$ appearing as a parameter. Recalling (1.12), we may thus estimate

$$
\begin{aligned}
\sup _{y}\left\|G^{I}(x, t ; y)\right\|_{L^{2}(x)} & =\sum_{j} \sup _{y}\left\|\phi(\xi) e^{\lambda_{j}(\xi) t} q_{j}\left(\cdot, z_{1}\right) \tilde{q}_{j}^{*}\left(\cdot, y_{1}\right)\right\|_{L^{2}\left(\xi ; L^{2}\left(z_{1} \in[0, X]\right)\right)} \\
& \leq C \sum_{j} \sup _{y}\left\|\phi(\xi) e^{-\theta|\xi|^{2} t}\right\|_{L^{2}(\xi)}\left\|q_{j}\right\|_{L^{2}(0, X)}\left\|\tilde{q}_{j}\right\|_{L^{\infty}(0, X)} \\
& \leq C(1+t)^{-\frac{d}{4}}
\end{aligned}
$$

where we have used in a crucial way the boundedness of $\tilde{q}_{j}$; derivative bounds follow similarly. Finally, bounds for $2 \leq p \leq \infty$ follow by $L^{p}$-interpolation.

Remark 2.4. In obtaining the key $L^{2}$-estimate, we have used in an essential way the periodic structure of $q_{j}, \tilde{q}_{j}$. For, viewing $G^{I}$ as a general pseudodifferential expression rather than a Bloch-Fourier decomposition, we find that the smoothness of $q_{j}, \tilde{q}_{j}$ is not sufficient to apply standard $L^{2} \rightarrow L^{2}$ bounds of Hörmander, which require blowup in $\xi$ derivatives at less than the critical rate $|\xi|^{-1}$ found here; see, e.g., $[\mathrm{H}]$ for further discussion. Nor do the weighted energy estimate techniques used in [S1, S2, S3] apply here, as these also rely on the property of smoothness of $\lambda_{j}, q_{j}, \tilde{q}_{j}$ with respect to $\xi$ at the origin $\xi=0$. The lack of smoothness of the linearized dispersion relation at the origin is an essential technical difference separating the conservation law from the reaction diffusion case; see OZ4 for further discussion.

Remark 2.5. Underlying the above analysis, and also the technically rather different approach of [OZ2], is the fundamental relation

$$
G(x, t ; y)=\left(\frac{1}{2 \pi}\right)^{d} \int_{-\pi}^{\pi} \int_{\mathbb{R}^{d-1}} e^{i \xi \cdot(x-y)}\left[G_{\xi}\left(x_{1}, t ; y_{1}\right)\right] d \xi
$$


which, provided $\sigma\left(L_{\xi}\right)$ is semisimple, yields the simple formula

$$
G(x, t ; y)=\left(\frac{1}{2 \pi}\right)^{d} \int_{-\pi}^{\pi} \int_{\mathbb{R}^{d-1}} e^{i \xi \cdot(x-y)} \sum_{j} e^{\lambda_{j}(\xi) t} q_{j}\left(\xi, x_{1}\right) \tilde{q}_{j}\left(\xi, y_{1}\right)^{*} d \xi
$$

resembling that of the constant-coefficient case, where $\lambda_{j}$ runs through the spectrum of $L_{\xi}$. The basic idea in both cases is to separate off the principal part of the series involving small $\lambda_{j}(\xi)$ and estimate the remainder as a faster-decaying residual.

Corollary 2.6 ([OZ4]). Under assumptions (H1)-(H3) and (D1)-(D3), for all $p \geq 2, t \geq 0$,

$$
\left\|S^{I}(t) f\right\|_{L^{p}},\left\|\partial_{x} S^{I}(t) f\right\|_{L^{p}},\left\|S^{I}(t) \partial_{x} f\right\|_{L^{p}} \leq C(1+t)^{-\frac{d}{2}\left(1-\frac{1}{p}\right)}\|f\|_{L^{1}} .
$$

Proof. Immediate, from (2.9) and the triangle inequality, as, for example,

$$
\left\|S^{I}(t) f(\cdot)\right\|_{L^{p}}=\left\|\int_{\mathbb{R}^{d}} G^{I}(x, t ; y) f(y) d y\right\|_{L^{p}(x)} \leq \int_{\mathbb{R}^{d}} \sup _{y}\left\|G^{I}(\cdot, t ; y)\right\|_{L^{p}}|f(y)| d y .
$$

Proposition 2.1 ([OZ4]). Assuming (H1)-(H3), (D1)-(D3), for some $C>0$, all $t \geq 0$, $p \geq 2,0 \leq l \leq K$,

$$
\left\|S(t) \partial_{x}^{l} u_{0}\right\|_{L^{p}} \leq C t^{-\frac{l}{2}}(1+t)^{-\frac{d}{2}\left(\frac{1}{2}-\frac{1}{p}\right)+\frac{l}{2}} t^{-\frac{d}{4}-\frac{l}{2}}\left\|u_{0}\right\|_{L^{1} \cap L^{2}} .
$$

Proof. Immediate, from (2.4) and (2.13).

\subsection{Additional estimates}

Lemma 2.7. Assuming (H1)-(H3), (D1)-(D3), for all $t \geq 0,0 \leq l \leq K$,

$$
\left\|\partial_{x}^{l} S^{I}(t) f\right\|_{L^{p}(x)},\left\|S^{I}(t) \partial_{x}^{l} f\right\|_{L^{p}(x)} \leq C(1+t)^{-\frac{d}{2}(1 / 2-1 / p)}\|f\|_{L^{2}(x)} .
$$

Proof. From boundedness of the spectral projections $P_{j}(\xi)=q_{j}\left\langle\tilde{q}_{j}, \cdot\right\rangle$ in $L^{2}[0, X]$ and their derivatives, another consequence of first-order splitting of eigenvalues $\lambda_{j}(\xi)$ at the origin, we obtain boundedness of $\phi(\xi) P(\xi) e^{L_{\xi} t}$ and thus, by (1.12), the global bounds

$$
\left\|\partial_{x}^{l} S^{I}(t) f\right\|_{L^{2}(x)},\left\|S^{I}(t) \partial_{x}^{l} f\right\|_{L^{2}(x)} \leq C\|f\|_{L^{2}(x)},
$$

for all $t \geq 0$, yielding the result for $p=2$. Moreover, by boundedness of $\tilde{q}, q$ in all $L^{p}\left(x_{1}\right)$, we have

$$
\left|\phi(\xi) P(\xi) e^{L_{\xi} t} \hat{f}(\xi, \cdot)\right|_{L^{\infty}\left(x_{1}\right)} \leq C e^{-\theta|\xi|^{2} t}|P(\xi) \hat{f}(\xi, \cdot)|_{L^{\infty}\left(x_{1}\right)} \leq C e^{-\theta|\xi|^{2} t}|\hat{f}(\xi, \cdot)|_{L^{2}\left(x_{1}\right)},
$$


$C, \theta>0$, yielding by $S^{I} f=\left(\frac{1}{2 \pi}\right)^{d} \int_{-\pi}^{\pi} \int_{\mathbb{R}^{d-1}} e^{i \xi \cdot x} \phi(\xi) P(\xi) e^{L_{\xi} t} \hat{f}\left(\xi, x_{1}\right) d \xi_{1} d \tilde{\xi}$ the bound

$$
\begin{aligned}
\left\|S^{I}(t) f\right\|_{L^{\infty}(x)} & \leq\left(\frac{1}{2 \pi}\right)^{d} \int_{-\pi}^{\pi} \int_{\mathbb{R}^{d-1}}\left|\phi(\xi) P(\xi) e^{L_{\xi} t} \hat{f}(\xi, \cdot)\right|_{L^{\infty}\left(x_{1}\right)} d \xi_{1} d \tilde{\xi} \\
& \leq\left(\frac{1}{2 \pi}\right)^{d} \int_{-\pi}^{\pi} \int_{\mathbb{R}^{d-1}} C \phi(\xi) e^{-\theta|\xi|^{2} t}|\hat{f}(\xi, \cdot)|_{L^{2}\left(x_{1}\right)} d \xi_{1} d \tilde{\xi} \\
& \leq C\left|\phi(\xi) e^{-\theta|\xi|^{2} t}\right|_{L^{2}(\xi)}|\hat{f}|_{L^{2}\left(\xi, x_{1}\right)} \\
& =C(1+t)^{-\frac{d}{4}}\|f\|_{L^{2}([0, X])},
\end{aligned}
$$

yielding the result for $p=\infty, l=0$. The result for $p=\infty, 1 \leq l \leq K$ follows by a similar argument. The result for general $2 \leq p \leq \infty$ then follows by $L^{p}$ interpolation between $p=2$ and $p=\infty$.

By Riesz-Thorin interpolation between (2.15) and (2.13), we obtain the following, apparently sharp bounds between various $L^{q}$ and $L^{p} 2$

Corollary 2.8. Assuming (HO)-(H3) and (D1)-(D3), for all $1 \leq q \leq 2 \leq p$, $t \geq 0$, $0 \leq l \leq K$,

$$
\left\|\partial_{x}^{l} S^{I}(t) f\right\|_{L^{p}},\left\|S^{I}(t) \partial_{x}^{l} f\right\|_{L^{p}} \leq C(1+t)^{-\frac{d}{2}\left(\frac{1}{q}-\frac{1}{p}\right)}\|f\|_{L^{q}}
$$

Proposition 2.2. Assuming (H1)-(H3), (D1)-(D3), for some $C>0$, all $t \geq 0,1 \leq q \leq$ $2 \leq p$, and $0 \leq l \leq K$,

$$
\left\|S(t) \partial_{x}^{l} u_{0}\right\|_{L^{p}} \leq C(1+t)^{-\frac{d}{2}\left(\frac{1}{2}-\frac{1}{p}\right)+\frac{l}{2}} t^{-\frac{d}{2}\left(\frac{1}{q}-\frac{1}{2}\right)-\frac{l}{2}}\left\|u_{0}\right\|_{L^{q} \cap L^{2}} .
$$

Proof. Immediate, from (2.4) and (2.8).

\section{$3 \quad$ Refined linearized estimates}

The bounds of Proposition 2.1 are sufficient to establish nonlinear stability and asymptotic behavior in dimensions $d \geq 3$, as shown in OZ4. However, they are not sufficient in the critical dimensions $d=1,2$; see Remark 1, Section 7 of [OZ4]. Comparison with standard diffusive stability arguments as in [Z7] show that this is due to the fact that the full solution operator $\left|S(t) \partial_{x}\right|$ decays no faster than $S(t)$, or, equivalently, $G_{y}$ no faster than $G$.

Following the basic strategy introduced in [ZH, ZZ1, MaZ2, MaZ4] in the context of viscous shock waves, we now perform a refined linearized estimate separating slower-decaying translational modes from a faster-decaying "good" part of the solution operator. This will be used in Section 4 in combination with certain nonlinear cancellation estimates to show convergence to the modulated approximation (1.1) at a faster rate sufficient to close the nonlinear iteration.

The key to this decomposition is the following observation.

\footnotetext{
${ }^{2}$ The inclusion of general $p \geq 2$ in Lemma 2.7 repairs an omission in [OZ4, where the bounds (2.8) were stated but not used.
} 
Lemma 3.1. Assuming (H1)-(H3), (D1)-(D3), let $\lambda_{j}(\xi /|\xi|, \xi), q_{j}(\xi /|\xi|, \xi, \cdot), \tilde{q}_{j}(\xi /|\xi|, \xi, \cdot)$ denote the eigenvalues and associated right and left eigenfunctions of $L_{\xi}$, with $q_{j}, \tilde{q}_{j}$ smooth functions of $\xi /|\xi|$ and $|\xi|$ as noted in Prop. 2.2. Then, without loss of generality, $q_{1}(\omega, 0, \cdot) \equiv$ $\bar{u}^{\prime}$, while $\tilde{q}_{j}(\omega, 0, \cdot)$ for $j \neq 1$ are constant functions depending only on angle $\omega=\xi /|\xi|$.

Proof. Expanding $L_{\xi}=L_{0}+|\xi| L_{\xi /|\xi|}^{1}+|\xi|^{2} L_{\xi /|\xi|}^{2}$ as in the introduction, consider the continuous family of spectral perturbation problems in $|\xi|$ indexed by angle $\omega=\xi /|\xi|$. Then, both facts follow by standard perturbation theory [K] using the observations that $\bar{u}^{\prime}$ is in the right kernel of $L_{0}$ and constant functions $c$ are in the left kernel of $L_{0}$, with

$$
\left.\left\langle c, L^{1} \bar{u}^{\prime}\right\rangle=\left\langle c,\left(\omega_{1}\left(2 \partial_{x_{1}}-A_{1}\right)-\sum_{j \neq 1} \omega_{j} A_{j}\right)\right) \bar{u}^{\prime}\right\rangle=\left\langle c, \omega_{1} \partial_{x_{1}}^{2} \bar{u}-\sum_{j \neq 1} \omega_{j} \partial_{x_{1}} f^{j}(\bar{u})\right\rangle \equiv 0,
$$

where $\langle\cdot, \cdot\rangle$ denotes $L^{2}\left(x_{1}\right)$ inner product on the interval $x_{1} \in[0, X]$, that the dimension of ker $L_{0}$ by assumption is $(n+1)$, so that the orthogonal complement of $\bar{u}^{\prime}$ in $\operatorname{Ker} L_{0}$ is dimension $n$ so exactly the set of constant functions, and that by (H3) the functions $q_{j}(\omega, 0, \cdot)$ and $\tilde{q}_{j}(\omega, 0)$ are right and left eigenfunctions of $\left.\Pi_{0} L^{1}\right|_{\operatorname{ker} L_{0}}\left(\Pi_{0}\right.$ as earlier denoting the zero eigenprojection associated with $\left.L_{0}\right)$.

Remark 3.2. The key observation of Lemma 3.1 can be motivated by the form of the Whitham averaged system (1.2). For, recalling (Section 1.3) that (D3) implies that speed $s$ is stationary to first order at $\bar{u}$ along the manifold of nearby periodic solutions, we find that the last equation of (1.2) reduces to $\left(\nabla_{x} \Psi\right)_{t}=0$, i.e., the equation for the translational variation $\Psi$ decouples from the equations for variations in other modes. This corresponds heuristically to the fact derived above that the translational mode $\bar{u}^{\prime}\left(x_{1}\right)$ decouples in the first-order eigenfunction expansion.

Corollary 3.1. Under assumptions (H1)-(H3), (D1)-(D3), the Green function $G(x, t ; y)$ of (1.7) decomposes as $G=E+\tilde{G}$,

$$
E=\bar{u}^{\prime}(x) e(x, t ; y)
$$

where, for some $C>0$, all $t>0,1 \leq q \leq 2 \leq p \leq \infty, 0 \leq j, k, l, j+l \leq K, 1 \leq r \leq 2$,

$$
\begin{aligned}
\left|\int_{-\infty}^{+\infty} \tilde{G}(x, t ; y) f(y) d y\right|_{L^{p}(x)} \leq & C(1+t)^{-\frac{d}{2}(1 / 2-1 / p)} t^{-\frac{1}{2}(1 / q-1 / 2)}|f|_{L^{q} \cap L^{2}}, \\
\left|\int_{-\infty}^{+\infty} \partial_{y}^{r} \tilde{G}(x, t ; y) f(y) d y\right|_{L^{p}(x)} \leq & C(1+t)^{-\frac{d}{2}(1 / 2-1 / p)-\frac{1}{2}+\frac{r}{2}} \\
& \times t^{-\frac{d}{2}(1 / q-1 / 2)-\frac{r}{2}}|f|_{L^{q} \cap L^{2}}, \\
\left|\int_{-\infty}^{+\infty} \partial_{t}^{r} \tilde{G}(x, t ; y) f(y) d y\right|_{L^{p}(x)} \leq & C(1+t)^{-\frac{d}{2}(1 / 2-1 / p)-\frac{1}{2}+r} \\
& \times t^{-\frac{d}{2}(1 / q-1 / 2)-r}|f|_{L^{q} \cap L^{2}} .
\end{aligned}
$$




$$
\left|\int_{-\infty}^{+\infty} \partial_{x}^{j} \partial_{t}^{k} \partial_{y}^{l} e(x, t ; y) f(y) d y\right|_{L^{p}} \leq(1+t)^{-\frac{d}{2}(1 / q-1 / p)-\frac{(j+k)}{2}}|f|_{L^{q}} .
$$

Moreover, $e(x, t ; y) \equiv 0$ for $t \leq 1$.

Proof. We first treat the simpler case $q=1$. Recalling that

$$
G^{I}(x, t ; y)=\left(\frac{1}{2 \pi}\right)^{d} \int_{\mathbb{R}^{d}} e^{i \xi \cdot(x-y)} \phi(\xi) \sum_{j=1}^{n+1} e^{\lambda_{j}(\xi) t} q_{j}\left(\xi, x_{1}\right) \tilde{q}_{j}\left(\xi, y_{1}\right)^{*} d \xi
$$

define

$$
\tilde{e}(x, t ; y)=\left(\frac{1}{2 \pi}\right)^{d} \int_{\mathbb{R}^{d}} e^{i \xi \cdot(x-y)} \phi(\xi) e^{\lambda_{1}(\xi) t} \tilde{q}_{1}\left(\xi, y_{1}\right)^{*} d \xi
$$

so that

$$
\begin{aligned}
G^{I}(x, t ; y)-\bar{u}^{\prime}\left(x_{1}\right) \tilde{e}(x, t ; y) & =\left(\frac{1}{2 \pi}\right)^{d} \int_{\mathbb{R}^{d}} e^{i \xi \cdot(x-y)} \phi(\xi) \sum_{j=2}^{n+1} e^{\lambda_{j}(\xi) t} q_{j}\left(\xi /|\xi|, 0, x_{1}\right) \tilde{q}_{j}\left(\xi, y_{1}\right)^{*} d \xi \\
& +\left(\frac{1}{2 \pi}\right)^{d} \int_{\mathbb{R}^{d}} \sum_{j=1}^{n+1} e^{i \xi \cdot(x-y)} \phi(\xi) e^{\lambda_{j}(\xi) t} O(|\xi|) d \xi
\end{aligned}
$$

Noting, by Lemma 3.1, that $\partial_{y} \tilde{q}(\omega, 0, y) \equiv$ constant for $j \neq 1$, we have therefore

$$
\partial_{y}^{r}\left(G^{I}(x, t ; y)-\bar{u}^{\prime}\left(x_{1}\right) \tilde{e}(x, t ; y)\right)=\left(\frac{1}{2 \pi}\right)^{d} \int_{\mathbb{R}^{d}} e^{i \xi \cdot(x-y)} \phi(\xi) \sum_{j=1}^{n+1} e^{\lambda_{j}(\xi) t} O(|\xi|) d \xi,
$$

which readily gives

$$
\left|\partial_{y}^{r}\left(G^{I}(x, t ; y)-\bar{u}^{\prime}\left(x_{1}\right) \tilde{e}(x, t ; y)\right)\right|_{L^{p}} \leq C(1+t)^{-\frac{d}{2}(1-1 / p)-\frac{1}{2}}
$$

$p \geq 2$, by the same argument used to prove (2.9), and similarly

$$
\left|\partial_{t}^{r}\left(G^{I}(x, t ; y)-\bar{u}^{\prime}\left(x_{1}\right) \tilde{e}(x, t ; y)\right)\right|_{L^{p}} \leq c(1+t)^{-\frac{d}{2}(1-1 / p)-\frac{1}{2}}
$$

These yield (3.2) by the triangle inequality.

Defining $e(x, t ; y):=\chi(t) \tilde{e}(x, t ; y)$, where $\chi$ is a smooth cutoff function such that $\chi(t) \equiv 1$ for $t \geq 2$ and $\chi(t) \equiv 0$ for $t \leq 1$, and setting $\tilde{G}:=G-\bar{u}^{\prime}\left(x_{1}\right) e(x, t ; y)$, we readily obtain the estimates (3.2) by combining (3.9) with bound (2.4) on $G^{I I}$. Bounds (3.3) follow from (3.5) by the argument used to prove (2.9), together with the observation that $x$ - or $t$-derivatives bring down factors of $|\xi|$, followed again by an application of the triangle inequality.

The cases $1 \leq q \leq 2$ follow similarly, by the arguments used to prove (2.15) and (2.8). 
Remark 3.3. Despite their apparent complexity, the above bounds may be recognized as essentially just the standard diffusive bounds satisfied for the heat equation [Z7]. For dimension $d=1$, it may be shown using pointwise techniques as in [OZ2] that the bounds of Corollary 3.1 extend to all $1 \leq q \leq p \leq \infty$.

Note the strong analogy between the Green function decomposition of Corollary 3.1 and that of [MaZ3, Z4] in the viscous shock case. We pursue this analogy further in the nonlinear analysis of the following sections, combining the "instantaneous tracking" strategy of [ZH, Z1, Z4, Z7, MaZ2, MaZ4] with a type of cancellation estimate introduced in [HoZ].

\section{Nonlinear stability in dimension one}

For clarity, we carry out the nonlinear stability analysis in detail in the most difficult, one-dimensional, case, indicating afterward by a few brief remarks the extension to $d=2$. Hereafter, take $x \in \mathbb{R}^{1}$, dropping the indices on $f^{j}$ and $x_{j}$ and writing $u_{t}+f(u)_{x}=u_{x x}$.

\subsection{Nonlinear perturbation equations}

Given a solution $\tilde{u}(x, t)$ of (1.4), define the nonlinear perturbation variable

$$
v=u-\bar{u}=\tilde{u}(x+\psi(x, t))-\bar{u}(x),
$$

where

$$
u(x, t):=\tilde{u}(x+\psi(x, t))
$$

and $\psi: \mathbb{R} \times \mathbb{R} \rightarrow \mathbb{R}$ is to be chosen later.

Lemma 4.1. For $v$, $u$ as in (4.1), (4.2),

$$
u_{t}+f(u)_{x}-u_{x x}=\left(\partial_{t}-L\right) \bar{u}^{\prime}\left(x_{1}\right) \psi(x, t)+\partial_{x} R+\left(\partial_{t}+\partial_{x}^{2}\right) S
$$

where

$$
R:=v \psi_{t}+v \psi_{x x}+\left(\bar{u}_{x}+v_{x}\right) \frac{\psi_{x}^{2}}{1+\psi_{x}}=O\left(|v|\left(\left|\psi_{t}\right|+\left|\psi_{x x}\right|\right)+\left(\frac{\left|\bar{u}_{x}\right|+\left|v_{x}\right|}{1-\left|\psi_{x}\right|}\right)\left|\psi_{x}\right|^{2}\right)
$$

and

$$
S:=-v \psi_{x}=O\left(|v|\left(\left|\psi_{x}\right|\right) .\right.
$$

Proof. To begin, notice from the definition of $u$ in (4.2) we have by a straightforward computation

$$
\begin{aligned}
u_{t}(x, t) & =\tilde{u}_{x}(x+\psi(x, t), t) \psi_{t}(x, t)+\tilde{u}_{t}(x+\psi, t) \\
f(u(x, t))_{x} & =d f(\tilde{u}(x+\psi(x, t), t)) \tilde{u}_{x}(x+\psi, t) \cdot\left(1+\psi_{x}(x, t)\right)
\end{aligned}
$$


and

$$
\begin{aligned}
u_{x x}(x, t) & =\left(\tilde{u}_{x}(x+\psi(x, t), t) \cdot\left(1+\psi_{x}(x, t)\right)\right)_{x} \\
& =\tilde{u}_{x x}(x+\psi(x, t), t) \cdot\left(1+\psi_{x}(x, t)\right)+\left(\tilde{u}_{x}(x+\psi(x, t), t) \cdot \psi_{x}(x, t)\right)_{x} .
\end{aligned}
$$

Using the fact that $\tilde{u}_{t}+d f(\tilde{u}) \tilde{u}_{x}-\tilde{u}_{x x}=0$, it follows that

$$
\begin{aligned}
u_{t}+f(u)_{x}-u_{x x} & =\tilde{u}_{x} \psi_{t}+d f(\tilde{u}) \tilde{u}_{x} \psi_{x}-\tilde{u}_{x x} \psi_{x}-\left(\tilde{u}_{x} \psi_{x}\right)_{x} \\
& =\tilde{u}_{x} \psi_{t}-\tilde{u}_{t} \psi_{x}-\left(\tilde{u}_{x} \psi_{x}\right)_{x}
\end{aligned}
$$

where it is understood that derivatives of $\tilde{u}$ appearing on the righthand side are evaluated at $(x+\psi(x, t), t)$. Moreover, by another direct calculation, using the fact that $L\left(\bar{u}^{\prime}(x)\right)=0$ by translation invariance, we have

$$
\left(\partial_{t}-L\right) \bar{u}^{\prime}(x) \psi=\bar{u}_{x} \psi_{t}-\bar{u}_{t} \psi_{x}-\left(\bar{u}_{x} \psi_{x}\right)_{x} .
$$

Subtracting, and using the facts that, by differentiation of $(\bar{u}+v)(x, t)=\tilde{u}(x+\psi, t)$,

$$
\begin{aligned}
\bar{u}_{x}+v_{x} & =\tilde{u}_{x}\left(1+\psi_{x}\right), \\
\bar{u}_{t}+v_{t} & =\tilde{u}_{t}+\tilde{u}_{x} \psi_{t},
\end{aligned}
$$

so that

$$
\begin{gathered}
\tilde{u}_{x}-\bar{u}_{x}-v_{x}=-\left(\bar{u}_{x}+v_{x}\right) \frac{\psi_{x}}{1+\psi_{x}}, \\
\tilde{u}_{t}-\bar{u}_{t}-v_{t}=-\left(\bar{u}_{x}+v_{x}\right) \frac{\psi_{t}}{1+\psi_{x}},
\end{gathered}
$$

we obtain

$$
u_{t}+f(u)_{x}-u_{x x}=\left(\partial_{t}-L\right) \bar{u}^{\prime}(x) \psi+v_{x} \psi_{t}-v_{t} \psi_{x}-\left(v_{x} \psi_{x}\right)_{x}+\left(\left(\bar{u}_{x}+v_{x}\right) \frac{\psi_{x}^{2}}{1+\psi_{x}}\right)_{x},
$$

yielding (4.3) by $v_{x} \psi_{t}-v_{t} \psi_{x}=\left(v \psi_{t}\right)_{x}-\left(v \psi_{x}\right)_{t}$ and $\left(v_{x} \psi_{x}\right)_{x}=\left(v \psi_{x}\right)_{x x}-\left(v \psi_{x x}\right)_{x}$.

Corollary 4.2. The nonlinear residual $v$ defined in (4.1) satisfies

$$
v_{t}-L v=\left(\partial_{t}-L\right) \bar{u}^{\prime}\left(x_{1}\right) \psi-Q_{x}+R_{x}+\left(\partial_{t}+\partial_{x}^{2}\right) S,
$$

where

$$
\begin{gathered}
Q:=f(\tilde{u}(x+\psi(x, t), t))-f(\bar{u}(x))-d f(\bar{u}(x)) v=\mathcal{O}\left(|v|^{2}\right), \\
R:=v \psi_{t}+v \psi_{x x}+\left(\bar{u}_{x}+v_{x}\right) \frac{\psi_{x}^{2}}{1+\psi_{x}}
\end{gathered}
$$

and

$$
S:=-v \psi_{x}=O\left(|v|\left(\left|\psi_{x}\right|\right) .\right.
$$

Proof. Taylor expansion comparing (4.3) and $\bar{u}_{t}+f(\bar{u})_{x}-\bar{u}_{x x}=0$. 


\subsection{Cancellation estimate}

Our strategy in writing (4.7) is motivated by the following basic cancellation principle.

Proposition 4.3 ([으] $)$. For any $f(y, s) \in L^{p} \cap C^{2}$ with $f(y, 0) \equiv 0$, there holds

$$
\int_{0}^{t} \int G(x, t-s ; y)\left(\partial_{s}-L_{y}\right) f(y, s) d y d s=f(x, t) .
$$

Proof. Integrating the left hand side by parts, we obtain

$$
\int G(x, 0 ; y) f(y, t) d y-\int G(x, t ; y) f(y, 0) d y+\int_{0}^{t} \int\left(\partial_{t}-L_{y}\right)^{*} G(x, t-s ; y) f(y, s) d y d s .
$$

Noting that, by duality,

$$
\left(\partial_{t}-L_{y}\right)^{*} G(x, t-s ; y)=\delta(x-y) \delta(t-s),
$$

$\delta(\cdot)$ here denoting the Dirac delta-distribution, we find that the third term on the righthand side vanishes in (4.12), while, because $G(x, 0 ; y)=\delta(x-y)$, the first term is simply $f(x, t)$. The second term vanishes by $f(y, 0) \equiv 0$.

Remark 4.1. For $\psi=\psi(t)$, term $\left(\partial_{t}-L\right) \bar{u}^{\prime} \psi$ in (4.7) reduces to the term $\dot{\psi}(t) \bar{u}^{\prime}(x)$ appearing in the shock wave case [ZH, ZZ1, ZZ4, Z7, MaZ2, MaZ4].

\subsection{Nonlinear damping estimate}

Proposition 4.2. Let $v_{0} \in H^{K}$ (K as in (H1)), and suppose that for $0 \leq t \leq T$, the $H^{K}$ norm of $v$ and the $H^{K}(x, t)$ norms of $\psi_{t}$ and $\psi_{x}$ remain bounded by a sufficiently small constant. There are then constants $\theta_{1,2}>0$ so that, for all $0 \leq t \leq T$,

$$
|v(t)|_{H^{K}}^{2} \leq C e^{-\theta_{1} t}|v(0)|_{H^{K}}^{2}+C \int_{0}^{t} e^{-\theta_{2}(t-s)}\left(|v|_{L^{2}}^{2}+\left|\left(\psi_{t}, \psi_{x}\right)\right|_{H^{K}(x, t)}^{2}\right)(s) d s .
$$

Proof. Subtracting from the equation (4.4) for $u$ the equation for $\bar{u}$, we may write the nonlinear perturbation equation as

$$
v_{t}+(d f(\bar{u}) v)_{x}-v_{x x}=Q(v)_{x}+\tilde{u}_{x} \psi_{t}-\tilde{u}_{t} \psi_{x}-\left(\tilde{u}_{x} \psi_{x}\right)_{x}
$$

where it is understood that derivatives of $\tilde{u}$ appearing on the righthand side are evaluated at $(x+\psi(x, t), t)$. Using (4.6) to replace $\tilde{u}_{x}$ and $\tilde{u}_{t}$ respectively by $\bar{u}_{x}+v_{x}-\left(\bar{u}_{x}+v_{x}\right) \frac{\psi_{x}}{1+\psi_{x}}$ and $\bar{u}_{t}+v_{t}-\left(\bar{u}_{x}+v_{x}\right) \frac{\psi_{t}}{1+\psi_{x}}$, and moving the resulting $v_{t} \psi_{x}$ term to the lefthand side of (4.14), we obtain

$$
\begin{aligned}
\left(1+\psi_{x}\right) v_{t}-v_{x x}= & -(d f(\bar{u}) v)_{x}+Q(v)_{x}+\bar{u}_{x} \psi_{t} \\
& -\left(\left(\bar{u}_{x}+v_{x}\right) \psi_{x}\right)_{x}+\left(\left(\bar{u}_{x}+v_{x}\right) \frac{\psi_{x}^{2}}{1+\psi_{x}}\right)_{x} .
\end{aligned}
$$


Taking the $L^{2}$ inner product in $x$ of $\sum_{j=0}^{K} \frac{\partial_{x}^{2 j} v}{1+\psi_{x}}$ against (4.15), integrating by parts, and rearranging the resulting terms, we arrive at the inequality

$$
\partial_{t}|v|_{H^{K}}^{2}(t) \leq-\theta\left|\partial_{x}^{K+1} v\right|_{L^{2}}^{2}+C\left(|v|_{H^{K}}^{2}+\left|\left(\psi_{t}, \psi_{x}\right)\right|_{H^{K}(x, t)}^{2}\right),
$$

for some $\theta>0, C>0$, so long as $|\tilde{u}|_{H^{K}}$ remains bounded, and $|v|_{H^{K}}$ and $\left|\left(\psi_{t}, \psi_{x}\right)\right|_{H^{K}(x, t)}$ remain sufficiently small. Using the Sobolev interpolation $|v|_{H^{K}}^{2} \leq\left|\partial_{x}^{K+1} v\right|_{L^{2}}^{2}+\tilde{C}|v|_{L^{2}}^{2}$ for $\tilde{C}>0$ sufficiently large, we obtain $\partial_{t}|v|_{H^{K}}^{2}(t) \leq-\tilde{\theta}|v|_{H^{K}}^{2}+C\left(|v|_{L^{2}}^{2}+\left|\left(\psi_{t}, \psi_{x}\right)\right|_{H^{K}(x, t)}^{2}\right)$ from which (4.13) follows by Gronwall's inequality.

\subsection{Integral representation $/ \psi$-evolution scheme}

By Proposition 4.3, we have, applying Duhamel's principle to (4.7),

$$
\begin{aligned}
v(x, t)= & \int_{-\infty}^{\infty} G(x, t ; y) v_{0}(y) d y \\
& +\int_{0}^{t} \int_{-\infty}^{\infty} G(x, t-s ; y)\left(-Q_{y}+R_{x}+S_{t}+S_{y y}\right)(y, s) d y d s+\psi(t) \bar{u}^{\prime}(x) .
\end{aligned}
$$

Defining $\psi$ implicitly as

$$
\begin{aligned}
\psi(x, t)= & -\int_{-\infty}^{\infty} e(x, t ; y) u_{0}(y) d y \\
& -\int_{0}^{t} \int_{-\infty}^{+\infty} e(x, t-s ; y)\left(-Q_{y}+R_{x}+S_{t}+S_{y y}\right)(y, s) d y d s
\end{aligned}
$$

following [ZH, Z4, MaZ2, MaZ3, where $e$ is defined as in (3.1), and substituting in (4.16) the decomposition $G=\bar{u}^{\prime}(x) e+\tilde{G}$ of Corollary 3.1, we obtain the integral representation

$$
\begin{aligned}
v(x, t)= & \int_{-\infty}^{\infty} \tilde{G}(x, t ; y) v_{0}(y) d y \\
& +\int_{0}^{t} \int_{-\infty}^{\infty} \tilde{G}(x, t-s ; y)\left(-Q_{y}+R_{x}+S_{t}+S_{y y}\right)(y, s) d y d s
\end{aligned}
$$

and, differentiating (4.17) with respect to $t$, and recalling that $e(x, s ; y) \equiv 0$ for $s \leq 1$,

$$
\begin{aligned}
\partial_{t}^{j} \partial_{x}^{k} \psi(x, t)= & -\int_{-\infty}^{\infty} \partial_{t}^{j} \partial_{x}^{k} e(x, t ; y) u_{0}(y) d y \\
& -\int_{0}^{t} \int_{-\infty}^{+\infty} \partial_{t}^{j} \partial_{x}^{k} e(x, t-s ; y)\left(-Q_{y}+R_{x}+S_{t}+S_{y y}\right)(y, s) d y d s .
\end{aligned}
$$

Equations (4.18), (4.19) together form a complete system in the variables $\left(v, \partial_{t}^{j} \psi, \partial_{x}^{k} \psi\right)$, $0 \leq j \leq 1,0 \leq k \leq K$, from the solution of which we may afterward recover the shift $\psi$ via (4.17). From the original differential equation (4.7) together with (4.19), we readily obtain short-time existence and continuity with respect to $t$ of solutions $\left(v, \psi_{t}, \psi_{x}\right) \in H^{K}$ by a standard contraction-mapping argument based on (4.13), (4.17), and and (3.3). 


\subsection{Nonlinear iteration}

Associated with the solution $\left(u, \psi_{t}, \psi_{x}\right)$ of integral system (4.18)-(4.19), define

$$
\zeta(t):=\sup _{0 \leq s \leq t}\left|\left(v, \psi_{t}, \psi_{x}\right)\right|_{H^{K}}(s)(1+s)^{1 / 4} .
$$

Lemma 4.3. For all $t \geq 0$ for which $\zeta(t)$ is finite, some $C>0$, and $E_{0}:=\left|u_{0}\right|_{L^{1} \cap H^{K}}$,

$$
\zeta(t) \leq C\left(E_{0}+\zeta(t)^{2}\right) .
$$

Proof. By (4.9)-(4.10) and definition (4.20),

$$
|(Q, R, S)|_{L^{1} \cap L^{\infty}} \leq\left|\left(v, v_{x}, \psi_{t}, \psi_{x}\right)\right|_{L^{2}}^{2}+\left|\left(v, v_{x}, \psi_{t}, \psi_{x}\right)\right|_{L^{\infty}}^{2} \leq C \zeta(t)^{2}(1+t)^{-\frac{1}{2}},
$$

so long as $\left|\psi_{x}\right| \leq\left|\psi_{x}\right|_{H^{K}} \leq \zeta(t)$ remains small, and likewise (using the equation to bound $t$ derivatives in terms of $x$-derivatives of up to two orders)

$$
\left|\left(\partial_{t}+\partial_{x}^{2}\right) S\right|_{L^{1} \cap L^{\infty}} \leq\left|\left(v, \psi_{x}\right)\right|_{H^{2}}^{2}+\left|\left(v, \psi_{x}\right)\right|_{W^{2, \infty}}^{2} \leq C \zeta(t)^{2}(1+t)^{-\frac{1}{2}}
$$

Applying Corollary 3.1 with $q=1, d=1$ to representations (4.18)-(4.19), we obtain for any $2 \leq p<\infty$

$$
\begin{aligned}
|v(\cdot, t)|_{L^{p}(x) \leq} & C(1+t)^{-\frac{1}{2}(1-1 / p)} E_{0} \\
& +C \zeta(t)^{2} \int_{0}^{t}(1+t-s)^{-\frac{1}{2}(1 / 2-1 / p)}(t-s)^{-\frac{3}{4}}(1+s)^{-\frac{1}{2}} d s \\
\leq & C\left(E_{0}+\zeta(t)^{2}\right)(1+t)^{-\frac{1}{2}(1-1 / p)}
\end{aligned}
$$

and

$$
\begin{aligned}
\left|\left(\psi_{t}, \psi_{x}\right)(\cdot, t)\right|_{W^{K, p}} & \leq C(1+t)^{-\frac{1}{2}} E_{0}+C \zeta(t)^{2} \int_{0}^{t}(1+t-s)^{-\frac{1}{2}(1-1 / p)-1 / 2}(1+s)^{-\frac{1}{2}} d s \\
& \leq C\left(E_{0}+\zeta(t)^{2}\right)(1+t)^{-\frac{1}{2}(1-1 / p)} .
\end{aligned}
$$

Using (4.13) and (4.24)-(4.25), we obtain $|v(\cdot, t)|_{H^{K}(x)} \leq C\left(E_{0}+\zeta(t)^{2}\right)(1+t)^{-\frac{1}{4}}$. Combining this with (4.25), $p=2$, rearranging, and recalling definition (4.20), we obtain (4.3).

Proof of Theorem 1.1. By short-time $H^{K}$ existence theory, $\left\|\left(v, \psi_{t}, \psi_{x}\right)\right\|_{H^{K}}$ is continuous so long as it remains small, hence $\eta$ remains continuous so long as it remains small. By (4.3), therefore, it follows by continuous induction that $\eta(t) \leq 2 C \eta_{0}$ for $t \geq 0$, if $\eta_{0}<1 / 4 C$, yielding by (4.20) the result (1.15) for $p=2$. Applying (4.24) -(4.25), we obtain (1.15) for $2 \leq p \leq p_{*}$ for any $p_{*}<\infty$, with uniform constant $C$. Taking $p_{*}>4$ and estimating

$$
|Q|_{L^{2}},|R|_{L^{2}},|S|_{L^{2}}(t) \leq\left|\left(v, \psi_{t}, \psi_{x}\right)\right|_{L^{4}}^{2} \leq C E_{0}(1+t)^{-\frac{3}{4}}
$$


in place of the weaker (4.22), then applying Corollary 3.1 with $q=2, d=1$, we obtain finally (1.15) for $2 \leq p \leq \infty$, by a computation similar (4.24)-(4.25); we omit the details of this final bootstrap argument. Estimate (1.16) then follows using (3.3) with $q=d=1$, by

$$
|\psi(t)|_{L^{p}} \leq C E_{0}+C \zeta(t)^{2} \int_{0}^{t}(1+t-s)^{-\frac{1}{2}(1-1 / p)}(1+s)^{-\frac{1}{2}} d s \leq C(1+t)^{\frac{1}{2 p}}\left(E_{0}+\zeta(t)^{2}\right),
$$

together with the fact that $\tilde{u}(x, t)-\bar{u}(x)=v(x-\psi, t)+(\bar{u}(x)-\bar{u}(x-\psi)$, so that $|\tilde{u}(\cdot, t)-\bar{u}|$ is controlled by the sum of $|v|$ and $|\bar{u}(x)-\bar{u}(x-\psi)| \sim|\psi|$. This yields stability for $\left.|u-\bar{u}|_{L^{1} \cap H^{K}}\right|_{t=0}$ sufficiently small, as described in the final line of the theorem.

\section{Nonlinear stability in dimension two}

We now briefly sketch the extension to dimension $d=2$. Given a solution $\tilde{u}(x, t)$ of (1.4), define the nonlinear perturbation variable

$$
v=u-\bar{u}=\tilde{u}\left(x_{1}+\psi(x, t), x_{2}, t\right)-\bar{u}\left(x_{1}\right),
$$

where

$$
u(x, t):=\tilde{u}\left(x_{1}+\psi(x, t), t\right)
$$

and $\psi: \mathbb{R}^{d} \times \mathbb{R} \rightarrow \mathbb{R}$ is to be chosen later.

Lemma 5.1. For $v$, $u$ as in (5.2),

$$
u_{t}+\sum_{j=1}^{d} f^{j}(u)_{x_{j}}-\sum_{j=1}^{d} u_{x_{j} x_{j}}=\left(\partial_{t}-L\right) \bar{u}^{\prime}\left(x_{1}\right) \psi(x, t)+\sum_{j=1}^{d} \partial_{x_{j}} R_{j}+\partial_{t} S+T,
$$

where

$R_{j}=O\left(\left(\mid v, \psi_{t}, \psi_{x}\right)||\left(v, v_{x}, \psi_{t}, \psi_{x}\right) \mid\right), \quad S:=-v \psi_{x_{1}}=\left(|v|\left(\left|\psi_{x}\right|\right), \quad T:=O\left(\left|\psi_{x}\right|^{3}+\left|\left(v, \psi_{x}\right)\right|\left|\psi_{x x}\right|\right)\right.$.

Proof. Similarly as in the proof of Lemma 4.1, it follows by a straightforward computation Using the fact that $\tilde{u}_{t}+\sum_{j} d f^{j}(\tilde{u}) \tilde{u}_{x_{j}}-\sum_{j} \tilde{u}_{x_{j} x_{j}}=0$, it follows that

$$
\begin{aligned}
u_{t}+\sum_{j} d f^{j}(u) u_{x_{j}}-\sum_{j} u_{x_{j} x_{j}}= & \tilde{u}_{x_{1}} \psi_{t}-\tilde{u}_{t} \psi_{x_{1}}+\sum_{j \neq 1} d f^{j}(\tilde{u}) \tilde{u}_{x_{1}} \psi_{x_{j}} \\
& -\sum_{j \neq 1} \tilde{u}_{x_{j} x_{1}} \psi_{x_{j}}-\sum_{j}\left(\tilde{u}_{x_{1}} \psi_{x_{j}}\right)_{x_{j}},
\end{aligned}
$$

where it is understood that derivatives of $\tilde{u}$ appearing on the righthand side are evaluated at $(x+\psi(x, t), t)$. Moreover, by another direct calculation, using the fact that $L\left(\bar{u}^{\prime}\left(x_{1}\right)\right)=0$ by translation invariance, we have

$$
\left(\partial_{t}-L\right) \bar{u}^{\prime}\left(x_{1}\right) \psi=\bar{u}_{x_{1}} \psi_{t}-\bar{u}_{t} \psi_{x_{1}}+\sum_{j \neq 1} d f^{j}(\bar{u}) \bar{u}_{x_{1}} \psi_{x_{j}}-\sum_{j \neq 1} \bar{u}_{x_{j} x_{1}} \psi_{x_{j}}-\sum_{j}\left(\bar{u}_{x_{1}} \psi_{x_{j}}\right)_{x_{j}} .
$$


Subtracting, and using (4.5) and

$$
\begin{aligned}
\bar{u}_{x_{j}}+v_{x_{j}} & =\tilde{u}_{x_{j}}+\tilde{u}_{x_{1}} \psi_{x_{j}}, \\
\bar{u}_{t}+v_{t} & =\tilde{u}_{t}+\tilde{u}_{x_{1}} \psi_{t},
\end{aligned}
$$

so that

$$
\begin{aligned}
\tilde{u}_{x_{j}}-\bar{u}_{x_{j}}-v_{x_{j}} & =-\left(\bar{u}_{x_{1}}+v_{x_{1}}\right) \frac{\psi_{x_{j}}}{1+\psi_{x_{1}}}, \\
\tilde{u}_{t}-\bar{u}_{t}-v_{t} & =-\left(\bar{u}_{x_{1}}+v_{x_{1}}\right) \frac{\psi_{t}}{1+\psi_{x_{1}}},
\end{aligned}
$$

we obtain

$$
\begin{aligned}
u_{t}+\sum_{j} d f^{j}(u) u_{x_{j}}-\sum_{j} u_{x_{j} x_{j}}= & \left(\partial_{t}-L\right) \bar{u}^{\prime}\left(x_{1}\right) \psi+v_{x_{1}} \psi_{t}-v_{t} \psi_{x_{1}} \\
& +\sum_{j \neq 1}\left(d f^{j}(\tilde{u}) \tilde{u}_{x_{1}}-d f^{j}(\bar{u}) \bar{u}_{x_{1}}\right) \psi_{x_{j}} \\
& -\sum_{j \neq 1}\left(\tilde{u}_{x_{j} x_{1}}-\bar{u}_{x_{j} x_{1}}\right) \psi_{x_{j}}-\sum_{j}\left(\left(\tilde{u}_{x_{1}}-\bar{u}_{x_{1}}\right) \psi_{x_{j}}\right)_{x_{j}} .
\end{aligned}
$$

Using $v_{x_{1}} \psi_{t}-v_{t} \psi_{x_{1}}=\left(v \psi_{t}\right)_{x_{1}}-\left(v \psi_{x_{1}}\right)_{t}$,

$$
d f^{j}(\tilde{u}) \tilde{u}_{x_{1}}=f(u)_{x_{1}}-d f^{j}(\tilde{u}) \tilde{u}_{x_{1}} \psi_{x_{1}}=f(u)_{x_{1}}\left(1-\psi_{x}\right)-d f^{j}(\tilde{u}) \tilde{u}_{x_{1}} \psi_{x_{1}}^{2},
$$

and $\tilde{u}_{x_{j} x_{1}}=\left(\tilde{u}_{x_{j}}\right)_{x_{1}}-\tilde{u}_{x_{j} x_{1}} \psi_{x_{1}}=\left(\tilde{u}_{x_{j}}\right)_{x_{1}}\left(1-\psi_{x_{1}}\right)+\tilde{u}_{x_{j} x_{1}} \psi_{x_{1}}^{2}$, and rearranging, we obtain

$$
\begin{aligned}
u_{t}+\sum_{j} d f^{j}(u) u_{x_{j}}-\sum_{j} u_{x_{j} x_{j}}= & \left(\partial_{t}-L\right) \bar{u}^{\prime}\left(x_{1}\right) \psi+\left(v \psi_{t}\right)_{x_{1}}-\left(v \psi_{x_{1}}\right)_{t} \\
& \left.+\sum_{j \neq 1}\left(f^{j}(u)-f^{j}(\bar{u})\right)_{x_{1}}\right) \psi_{x_{j}} \\
& -\sum_{j \neq 1} f(u)_{x_{1}} \psi_{x_{1}} \psi_{x_{j}}-\sum_{j \neq 1} d f^{j}(\tilde{u}) \tilde{u}_{x_{1}} \psi_{x_{1}}^{2} \psi_{x_{j}} \\
& -\sum_{j \neq 1}\left(\tilde{u}_{x_{j}}-\bar{u}_{x_{j}}\right)_{x_{1}} \psi_{x_{j}}+\sum_{j \neq 1}\left(\tilde{u}_{x_{j}}\right)_{x_{1}} \psi_{x_{1}} \psi_{x_{j}} \\
& +\sum_{j \neq 1} \tilde{u}_{x_{j} x_{1}} \psi_{x_{1}}^{2} \psi_{x_{j}} \\
& -\sum_{j}\left(v_{x_{1}} \psi_{x_{1}}\right)_{x_{j}}-\sum_{j}\left(\left(\bar{u}_{x_{1}}+v_{x_{1}}\right) \frac{\psi_{x_{j}} \psi_{x_{1}}}{1+\psi_{x_{1}}}\right)_{x_{j}} .
\end{aligned}
$$

Noting that

$$
\left.\left(f^{j}(u)-f^{j}(\bar{u})\right)_{x_{1}}\right) \psi_{x_{j}}=\left(\left(f^{j}(u)-f^{j}(\bar{u}) \psi_{x_{j}}\right)_{x_{1}}-\left(f^{j}(u)-f^{j}(\bar{u})\right) \psi_{x_{j} x_{1}},\right.
$$




$$
f(u)_{x_{1}} \psi_{x_{1}} \psi_{x_{j}}=\left(f(u) \psi_{x_{1}} \psi_{x_{j}}\right)_{x_{1}}-f(u)\left(\psi_{x_{1}} \psi_{x_{j}}\right)_{x_{1}},
$$

and

$$
\left(\tilde{u}_{x_{j}}-\bar{u}_{x_{j}}\right)_{x_{1}} \psi_{x_{j}}=\left(\left(\tilde{u}_{x_{j}}-\bar{u}_{x_{j}}\right) \psi_{x_{j}}\right)_{x_{1}}-\left(\tilde{u}_{x_{j}}-\bar{u}_{x_{j}}\right) \psi_{x_{j} x_{1}},
$$

with $\left|f^{j}(u)-f^{j}(\bar{u})\right|=O(|v|)$ and $\left|\tilde{u}_{x_{j}}-\bar{u}_{x_{j}}\right|=O(|v|)$, we obtain the result

Proof of Theorem 1.2. The result of Lemma 5.1 is the only part of the analysis that differs essentially from that of the one-dimensional case. The cancellation and nonlinear damping arguments go through exactly as before to yield the analogs of Propositions 4.3 and (4.2). Likewise, we obtain a Duhamel representation analogous to (4.18)-(4.19), forming a closed system in variables $\left(v, \psi_{x}, \psi_{t}\right)$.

To obtain the analog of Lemma 4.3, completing the proof of nonlinear stability, we can carry out a somewhat simpler argument than in the one-dimensional case, using Corollary 3.1 with $d=2, q=2$ for all estimates, not only the final bootstrap argument, giving in place of (4.24) the estimate

$$
\begin{aligned}
& |v(\cdot, t)|_{L^{p}(x)} \leq C(1+t)^{-(1-1 / p)} E_{0}+C \zeta(t)^{2} \int_{0}^{t}(1+t-s)^{-(1 / 2-1 / p)}(t-s)^{-\frac{1}{2}}(1+s)^{-1} d s \\
& \leq C\left(E_{0}+\zeta(t)^{2}\right)(1+t)^{-(1-1 / p)} \\
& \left|\left(\psi_{x}, \psi_{t}\right)(\cdot, t)\right|_{L^{p}(x)} \leq \\
& \quad C(1+t)^{-(1-1 / p)-\frac{1}{2}} E_{0} \\
& \quad+C \zeta(t)^{2} \int_{0}^{t}(1+t-s)^{-(1 / 2-1 / p)}(t-s)^{-\frac{1}{2}}(1+s)^{-1} d s \\
& \leq C\left(E_{0}+\zeta(t)^{2}\right)(1+t)^{\varepsilon-(1-1 / p)-\frac{1}{2}}
\end{aligned}
$$

for divergence-form source terms, and

$$
\begin{aligned}
|v(\cdot, t)|_{L^{p}(x)} \leq & C \zeta(t)^{2} \int_{0}^{t}(1+t-s)^{-(1 / 2-1 / p)}(1+s)^{-\frac{3}{2}} d s \\
\leq & C\left(E_{0}+\zeta(t)^{2}\right)(1+t)^{-(1-1 / p)}, \\
\left|\left(\psi_{x}, \psi_{t}\right)(\cdot, t)\right|_{L^{p}(x)} \leq & C(1+t)^{-(1-1 / p)-\frac{1}{2}} E_{0} \\
& +C \zeta(t)^{2} \int_{0}^{t}(1+t-s)^{-(1 / 2-1 / p)}(t-s)^{-\frac{1}{2}}(1+s)^{-\frac{3}{2}} d s \\
\leq & C\left(E_{0}+\zeta(t)^{2}\right)(1+t)^{\varepsilon-(1-1 / p)-\frac{1}{2}}
\end{aligned}
$$

for faster-decaying nondivergence-form source terms.

We omit the details, which are entirely similar to, but substantially simpler than, those of the one-dimensional case. 


\section{References}

[G] R. Gardner, On the structure of the spectra of periodic traveling waves, J. Math. Pures Appl. 72 (1993), 415-439.

[GZ] R. Gardner and K. Zumbrun, The Gap Lemma and geometric criteria for instability of viscous shock profiles, Comm. Pure Appl. Math. 51 (1998), no. 7, 797-85.

[He] D. Henry, Geometric theory of semilinear parabolic equations, Lecture Notes in Mathematics, Springer-Verlag, Berlin (1981).

[HoZ] D. Hoff and K. Zumbrun Asymptotic behavior of multidimensional scalar viscous shock fronts, Indiana Univ. Math. Journal, Vol. 49, No. 2 (2000).

[H] I.L. Hwang, The $L^{2}$-boundedness of pseudodifferential operators, Trans. Amer. Math. Soc. 302 (1987) 55-76.

[JZ1] M. Johnson and K. Zumbrun, Rigorous Justification of the Whitham Modulation Equations for the Generalized Korteweg-de Vries Equation, preprint (2009).

[JZB] M. Johnson, K. Zumbrun, and J. Bronski, Bloch wave expansion vs. Whitham Modulation Equations for the Generalized Korteweg-de Vries Equation, in preparation.

[K] T. Kato, Perturbation theory for linear operators, Springer-Verlag, Berlin Heidelberg (1985).

[MaZ2] C. Mascia and K. Zumbrun, Stability of small-amplitude shock profiles of symmetric hyperbolic-parabolic systems, Comm. Pure Appl. Math. 57 (2004), no. 7, 841-876.

[MaZ3] C. Mascia and K. Zumbrun, Pointwise Green function bounds for shock profiles of systems with real viscosity. Arch. Ration. Mech. Anal. 169 (2003), no. 3, 177263.

[MaZ4] C. Mascia and K. Zumbrun, Stability of large-amplitude viscous shock profiles of hyperbolic-parabolic systems, Arch. Ration. Mech. Anal. 172 (2004), no. 1, 93-131.

[OZ1] M. Oh and K. Zumbrun, Stability of periodic solutions of viscous conservation laws with viscosity- 1. Analysis of the Evans function, Arch. Ration. Mech. Anal. 166 (2003), no. 2, 99-166.

[OZ2] M. Oh and K. Zumbrun, Stability of periodic solutions of viscous conservation laws with viscosity- Pointwise bounds on the Green function, Arch. Ration. Mech. Anal. 166 (2003), no. 2, 167-196. 
[OZ3] M. Oh, and K. Zumbrun, Low-frequency stability analysis of periodic travelingwave solutions of viscous conservation laws in several dimensions, Journal for Analysis and its Applications, 25 (2006), 1-21.

[OZ4] M. Oh, and K. Zumbrun, Stability and asymptotic behavior of traveling-wave solutions of viscous conservation laws in several dimensions, to appear, Arch. Ration. Mech. Anal.

[Pa] A. Pazy, Semigroups of linear operators and applications to partial differential equations, Applied Mathematical Sciences, 44, Springer-Verlag, New YorkBerlin, (1983) viii+279 pp. ISBN: 0-387-90845-5.

[S1] G. Schneider, Nonlinear diffusive stability of spatially periodic solutions-abstract theorem and higher space dimensions, Proceedings of the International Conference on Asymptotics in Nonlinear Diffusive Systems (Sendai, 1997), 159-167, Tohoku Math. Publ., 8, Tohoku Univ., Sendai, 1998.

[S2] G. Schneider, Diffusive stability of spatial periodic solutions of the SwiftHohenberg equation, (English. English summary) Comm. Math. Phys. 178 (1996), no. 3, 679-702.

[S3] G. Schneider, Nonlinear stability of Taylor vortices in infinite cylinders, Arch. Rat. Mech. Anal. 144 (1998) no. 2, 121-200.

[Se1] D. Serre, Spectral stability of periodic solutions of viscous conservation laws: Large wavelength analysis, Comm. Partial Differential Equations 30 (2005), no. $1-3,259-282$.

[Z1] K. Zumbrun, Refined wave-tracking and stability of viscous Lax shocks, Methods Appl. Anal. 7 (2000) 747-768.

[Z4] K. Zumbrun, Stability of large-amplitude shock waves of compressible NavierStokes equations, with an appendix by Helge Kristian Jenssen and Gregory Lyng, in Handbook of mathematical fluid dynamics. Vol. III, 311-533, North-Holland, Amsterdam, (2004).

[Z6] K. Zumbrun, Dynamical stability of phase transitions in the p-system with viscosity-capillarity, SIAM J. Appl. Math. 60 (2000), 1913-1929.

[Z7] K. Zumbrun, Instantaneous shock location and one-dimensional nonlinear stability of viscous shock waves, preprint (2009).

[ZH] K. Zumbrun and P. Howard, Pointwise semigroup methods and stability of viscous shock waves. Indiana Mathematics Journal V47 (1998), 741-871; Errata, Indiana Univ. Math. J. 51 (2002), no. 4, 1017-1021. 